\title{
ON THE ESSENTIAL NUMERICAL RANGE OF A GENERALIZED DERIVATION
}

\begin{abstract}
BOJAN MAGAJNA ${ }^{1}$
Abstract. Let $A$ and $B$ be bounded operators on Hilbert spaces $\mathscr{H}$ and $\mathscr{L}$, respectively. The essential numerical range of the operator $X \rightarrow A X-X B$, defined on the Hilbert-Schmidt class $\mathscr{C}^{2}(\mathscr{L}, \mathscr{H})$ is expressed in terms of the numerical and the essential numerical ranges of $A$ and $B$.
\end{abstract}

1. Introduction and preliminaries. A generalized derivation on the algebra $\mathscr{B}(\mathscr{H})$ of all bounded operators on a Hilbert space $\mathscr{H}$ is an operator on $\mathscr{B}(\mathscr{H})$ of the form

$$
X \rightarrow A X-X B, \quad X \in \mathscr{B}(\mathscr{H}),
$$

where $A$ and $B$ are fixed elements of $\mathscr{B}(\mathscr{H})$. In the past, generalized derivations and their restrictions to norm ideals in $\mathscr{B}(\mathscr{H})$ have been studied by many authors. Up to now their spectra and essential spectra have been characterized $[7,8,13]$, and their norms [16] and numerical ranges [12, 15] have been determined. For bounded operators on Hilbert spaces the concept of the essential numerical range plays an important role, and in this note the essential numerical range of the restriction of a generalized derivation to the Hilbert-Schmidt class will be determined.

Before stating the results we establish the notation. For every $T \in \mathscr{B}(\mathscr{H})$ the spatial numerical range, $W(T)$, is defined by

$$
W(T)=\{\langle T x, x\rangle ; x \in \mathscr{H},\|x\|=1\} .
$$

If $\mathscr{A}$ is a Banach algebra with unit 1 , then the algebra numerical range of an arbitrary element $T \in \mathscr{A}$ is defined by

$$
V(T)=\left\{f(T) ; f \in \mathscr{A}^{\prime},\|f\|=f(1)=1\right\} .
$$

Here, of course, $\mathscr{A}^{\prime}$ denotes the space of all continuous linear functionals on $\mathscr{A}$. It is well known that for a Hilbert space operator $T$ the algebra numerical range $V(T)$ of $T$ (considered as an element of the algebra $\mathscr{B}(\mathscr{H})$ ) is simply the closure of the spatial numerical range $W(T)$ (see [2]). The essential numerical range, $V_{e}(T)$, of an operator $T \in \mathscr{B}(\mathscr{H})$ is (by definition) the numerical range of the coset $T+\mathscr{K}(\mathscr{H})$ in the Calkin algebra $\mathscr{B}(\mathscr{H}) / \mathscr{K}(\mathscr{H})$, where $\mathscr{K}(\mathscr{H})$ is the ideal of all compact

Received by the editors May 2, 1985 and, in revised form, September 23, 1985 and November 25, 1985.

1980 Mathematics Subject Classification (1985 Revision). Primary 47B47; Secondary 47A12.

Key words and phrases. Generalized derivations, essential numerical range, Hilbert-Schmidt class.

${ }^{1}$ The results here form a part of the author's Ph.D. thesis. The author would like to express his gratitude to Professor I. Vidav for his guidance during the preparation of the thesis. 
operators on $\mathscr{H}$. (For more details about numerical ranges in Banach algebras, see [2 and 3], and for the essential numerical ranges in particular, see [6 and 3]. We need the following characterization of the essential numerical ranges, obtained by Fillmore, Stampfli, and Williams in [6]: For $T \in \mathscr{B}(\mathscr{H}), \lambda \in V_{e}(T)$ if and only if there exists an orthonormal sequence $\left(x_{n}\right)$ in $\mathscr{H}$ such that $\lambda=\lim \left\langle T x_{n}, x_{n}\right\rangle$.

The class of all Hilbert-Schmidt operators from a Hilbert space $\mathscr{L}$ to a Hilbert space $\mathscr{H}$ will be denoted by $\mathscr{C}^{2}(\mathscr{L}, \mathscr{H})$ and, of course, $\mathscr{C}^{2}(\mathscr{H})=\mathscr{C}^{2}(\mathscr{H}, \mathscr{H})$. (The reader is referred to [14] for the definition of the Hilbert-Schmidt class.) Recall that $\mathscr{C}^{2}(\mathscr{L}, \mathscr{H})$ is a Hilbert space and that $A X B \in \mathscr{C}^{2}(\mathscr{L}, \mathscr{H})$ for every $A \in$ $\mathscr{B}(\mathscr{H}), X \in \mathscr{C}^{2}(\mathscr{L}, \mathscr{H})$ and $B \in \mathscr{B}(\mathscr{L})$; in particular, the operator

$$
D_{A B}(X)=A X-X B, \quad X \in \mathscr{C}^{2}(\mathscr{L}, \mathscr{H}),
$$

is a bounded endomorphism of $\mathscr{C}^{2}(\mathscr{L}, \mathscr{H})$.

For any subset $V$ of $\mathbf{C}$ we denote by $\operatorname{co}[V]$ the convex hull of $V$.

2. The essential numerical range. The main result of this paper is the following

Theorem. Let $\mathscr{H}, \mathscr{L}$ be separable Hilbert spaces and $A \in \mathscr{B}(\mathscr{H}), B \in \mathscr{B}(\mathscr{L})$. Then

$$
V_{e}\left(D_{A B}\right)=\operatorname{co}\left[\left(V_{e}(A)-V(B)\right) \cup\left(V(A)-V_{e}(B)\right)\right] .
$$

(Here, e.g., $V_{e}(A)-V(B)$ denotes the set $\left\{\lambda-\mu ; \lambda \in V_{e}(A), \mu \in V(B)\right\}$.)

REMARK 1. The equality (1) is similar to the formula for the essential spectra of generalized derivations proved by Fialkow in [8], and as such, it is not unexpected, but the method of proof is quite different.

In the proof of this theorem it is convenient to exploit the language of tensor products. Recall $[17,4]$ that, as a Hilbert space, $\mathscr{C}^{2}(\mathscr{L}, \mathscr{H})$ is naturally isomorphic to the tensor product $\mathscr{H} \otimes \overline{\mathscr{L}}$, and that this isomorphism implements the unitary equivalence between the operators $D_{A B}$ and $A \otimes I_{\overline{\mathscr{L}}}+I_{\mathscr{H}} \otimes \bar{B}^{*}$. (Here $\overline{\mathscr{L}}$ denotes the Hilbert space opposite to $\mathscr{L}: \overline{\mathscr{L}}$ is the same set as $\mathscr{L}$, with the same addition, but multiplication by scalars is defined by $\lambda \circ x=\bar{\lambda} x(\lambda \in \mathbf{C}, x \in \mathscr{L})$, and the inner product is defined by $[x, y]=\langle y, x\rangle$. Every linear operator $B$ on $\mathscr{L}$ induces a linear operator $\bar{B}$ on $\overline{\mathscr{L}}$ by $\bar{B} x=B x, x \in \mathscr{L}$.) Since unitarily equivalent operators clearly have the same essential numerical range, the equality (1) is equivalent to

$$
V_{e}\left(A \otimes I_{\overline{\mathscr{L}}}-I_{\mathscr{H}} \otimes \bar{B}^{*}\right)=\operatorname{co}\left[\left(V_{e}(A)-V(B)\right) \cup\left(V(A)-V_{e}(B)\right)\right] .
$$

Replace now in the last equality $\mathscr{L}$ and $B$ with $\overline{\mathscr{L}}$ and $\bar{B}^{*}$ respectively and note that $\overline{\bar{L}}=\mathscr{L},\left(\overline{\bar{B}}^{*}\right)^{*}=B, V\left(\bar{B}^{*}\right)=V(B)$ and $V_{e}\left(\bar{B}^{*}\right)=V_{e}(B)$. (One way to verify the relations $V\left(\bar{B}^{*}\right)=V(B)$ and $V_{e}\left(\bar{B}^{*}\right)=V_{e}(B)$ is through the observation that the map $T \rightarrow \bar{T}^{*}$ is a linear isometry of $\mathscr{B}(\mathscr{L})$ onto $\mathscr{B}(\overline{\mathscr{L}})$ which maps the identity operator $I_{\mathscr{L}}$ to $I_{\overline{\mathscr{L}}}$ and the ideal $\mathscr{K}(\mathscr{L})$ to $\mathscr{K}(\overline{\mathscr{L}})$.) We obtain in this way an equivalent equality. Thus, to prove the theorem, it suffices to prove the two inclusions

$$
\begin{aligned}
& \operatorname{co}\left[\left(V_{e}(A)-V(B)\right) \cup\left(V(A)-V_{e}(B)\right)\right] \subseteq V_{e}\left(A \otimes I_{\mathscr{L}}-I_{\mathscr{H}} \otimes B\right), \\
& V_{e}\left(A \otimes I_{\mathscr{L}}-I_{\mathscr{H}} \otimes B\right) \subseteq \operatorname{co}\left[\left(V_{e}(A)-V(B)\right) \cup\left(V(A)-V_{e}(B)\right)\right],
\end{aligned}
$$

where $\mathscr{H}$ and $\mathscr{L}$ are any separable Hilbert spaces and $A \in \mathscr{B}(\mathscr{H}), B \in \mathscr{B}(\mathscr{L})$. 
Proof of the inclusion (2). Put $D=A \otimes I_{\mathscr{L}}-I_{\mathscr{H}} \otimes B$. Since $V_{e}(D)$ is a convex compact set, and since $V(A)$ (respectively $V(B)$ ) is the closure of $W(A)$ (respectively $W(B)$ ), it suffices to prove that

$$
V_{e}(A)-W(B) \subseteq V_{e}(D) \text { and } W(A)-V_{e}(B) \subseteq V_{e}(D) .
$$

We shall prove only the first inclusion, for the proof of the second is similar.

Let $\lambda \in V_{e}(A), \mu \in W(B)$, let $\left(x_{n}\right)$ be an orthonormal sequence in $\mathscr{H}$ such that $\lim \left\langle A x_{n}, x_{n}\right\rangle=\lambda$, and let $y$ be a unit vector in $\mathscr{L}$ such that $\langle B y, y\rangle=\mu$. Then the sequence $\left(x_{n} \otimes y\right)$ in $\mathscr{H} \otimes \mathscr{L}$ is orthonormal, and we have

$$
\begin{aligned}
\left\langle D\left(x_{n} \otimes y\right), x_{n} \otimes y\right\rangle & =\left\langle A x_{n}, x_{n}\right\rangle\langle y, y\rangle-\left\langle x_{n}, x_{n}\right\rangle\langle B y, y\rangle \\
& =\left\langle A x_{n}, x_{n}\right\rangle-\langle B y, y\rangle .
\end{aligned}
$$

It follows that the sequence $\left(\left\langle D\left(x_{n} \otimes y\right), x_{n} \otimes y\right\rangle\right)$ converges to $\lambda-\mu$, hence $\lambda-\mu \in V_{e}(D)$.

REMARK 2. The inclusion corresponding to (2) holds also for generalized derivations on $\mathscr{B}(\mathscr{H})$, on norm ideals in $\mathscr{B}(\mathscr{H})$, and on irreducible $C^{*}$-subalgebras of $\mathscr{B}(\mathscr{H})$. In fact, if $\mathscr{A}$ is an irreducible $C^{*}$-subalgebra of $\mathscr{B}(\mathscr{H}), A, B \in \mathscr{A}$ and $D$ the generalized derivation $X \rightarrow A X-X B$ on $\mathscr{A}$, then one can prove the inclusion $V_{e}(A)-W(B) \subseteq V_{e}(D)$ by the following modification of the above proof. By the transitivity theorem [17, p. 93] there exists for each $n=1,2, \ldots$, an operator $U_{n} \in \mathscr{A}$ with norm 1 (in fact $U_{n}$ can be chosen to be unitary) such that $U_{n} y=x_{n}$. Now we can define a bounded linear functional $f$ on the algebra $\mathscr{B}(\mathscr{A})$ of all bounded operators on $\mathscr{A}$ by

$$
f(T)=\operatorname{Lim}\left(\left\langle T\left(U_{n}\right) y, x_{n}\right\rangle\right), \quad T \in \mathscr{B}(\mathscr{A}),
$$

where Lim is any Banach limit on the complex space $l^{\infty}[5$, p. 85]. Using the fact that the image of the unit ball of $\mathscr{A}$ by a compact operator has a finite $\varepsilon$-net for each $\varepsilon>0$, it is easy to see that $f$ annihilates the ideal $\mathscr{K}(\mathscr{A})$ of all compact operators on $\mathscr{A}$. Hence $f$ induces a functional $\tilde{f}$ on $\mathscr{B}(\mathscr{A}) / \mathscr{K}(\mathscr{A})$, and it is easy to verify that $\tilde{f}(1)=1=\|\tilde{f}\|$ and $\tilde{f}(D+\mathscr{K}(\mathscr{A}))=\lambda-\mu$. Thus $\lambda-\mu \in V_{e}(D)$.

REMARK 3. The author does not know whether the inclusion corresponding to (3) holds also for generalized derivations on $\mathscr{B}(\mathscr{H})$. In the proof of (3) we shall use the identities

$$
V(T \oplus S)=\operatorname{co}[V(T) \cup V(S)], \quad V_{e}(T \oplus S)=\operatorname{co}\left[V_{e}(T) \cup V_{e}(S)\right],
$$

that hold for arbitrary Hilbert space operators $T$ and $S$. The first identity is well known and can be proved for the the usual spatial numerical ranges by a direct computation, and then for the algebra numerical ranges by taking the closures. The second identity can be easily verified by using the following characterization of the essential numerical range [6]: for any Hilbert space operator $R \in \mathscr{B}(\mathscr{H}), \lambda \in V_{e}(R)$ if and only if there exists a sequence of unit vectors $x_{n}$ in $\mathscr{H}$ that converge weakly to 0 such that $\lambda=\lim \left\langle R x_{n}, x_{n}\right\rangle$. We shall apply these identities to operators on $\mathscr{C}^{2}(\mathscr{L}, \mathscr{H})$.

To prove the inclusion (3) we first need a simple lemma. For every subset $V$ of $\mathbf{C}$ and for every $\varepsilon>0$ put

$$
(V)_{\varepsilon}=\{\lambda \in \mathbf{C} ; \operatorname{dist} .(\lambda, V)<\varepsilon\} .
$$


LeMma 1. For each $T \in \mathscr{B}(\mathscr{H})$ and each $\varepsilon>0$ there exists a subspace $\mathscr{H}_{\varepsilon}$ of finite codimension in $\mathscr{H}$ such that the numerical range of the compression $T_{\varepsilon}$ of $T$ to $\mathscr{H}_{\varepsilon}$ is contained in the set $\left(V_{e}(T)\right)_{\varepsilon}$.

Proof. Note first that for each $\lambda \in V(T) \backslash V_{e}(T)$ there exists a subspace $\mathscr{H}_{\lambda}$ of finite codimension in $\mathscr{H}$ such that $\lambda \notin V\left(T_{\lambda}\right)$, where $T_{\lambda}$ denotes the compression of $T$ to $\mathscr{H}_{\lambda}$. For, in the opposite case one can construct inductively an orthonormal sequence $\left(x_{n}\right)$ in $\mathscr{H}$ such that $\lim \left\langle T x_{n}, x_{n}\right\rangle=\lambda$, but then we would have $\lambda \in V_{e}(T)$. Since $V\left(T_{\lambda}\right)$ is a closed set, there exists an open neighborhood $U_{\lambda}$ of $\lambda$ such that $U_{\lambda} \cap V\left(T_{\lambda}\right)=\varnothing$. Let $\left\{U_{\lambda_{1}}, \ldots, U_{\lambda_{n}}\right\}$ be a finite open covering of the compact set $V(T) \backslash\left(V_{e}(T)\right)_{\varepsilon}$ by such neighborhoods. Then the subspace

$$
\mathscr{H}_{\varepsilon}=\bigcap_{j=1}^{n} \mathscr{H}_{\lambda,}
$$

satisfies the requirements.

We shall prove the inclusion (3) first in the special case when $A$ and $B$ are quasidiagonal operators. (An operator $T \in \mathscr{B}(\mathscr{H})$ is quasidiagonal $[10,11])$ if and only if for every finite-dimensional subspace $\mathscr{H}_{0}$ of $\mathscr{H}$ and every $\varepsilon>0$ there exists a finite-dimensional subspace $\mathscr{H}_{1}$ of $\mathscr{H}_{\text {such that }} \mathscr{H}_{1}$ contains $\mathscr{H}_{0}$ and such that in the matrix representation of $T$ relative to the decomposition $\mathscr{H}=\mathscr{H}_{1} \oplus \mathscr{H}_{1}{ }^{\perp}$ the off-diagonal elements have norm less than $\varepsilon$. Later we shall need the fact that a compact perturbation of a quasidiagonal operator is quasidiagonal [11, p. 147].)

Proof OF THE INCLUSION (3) FOR QUASIDIAGONAL OPERATORS $A$ AND $B$. Let $\varepsilon>0$ be given. If $\mathscr{H}_{1}$ and $\mathscr{L}_{1}$ are finite-dimensional subspaces of $\mathscr{H}$ and $\mathscr{L}$, and if $\mathscr{H}_{2}=\mathscr{H}_{1}{ }^{\perp}, \mathscr{L}_{2}=\mathscr{L}_{1}{ }^{\perp}$, denote by $A_{i}$ and $B_{i}$ the compression of $A$ and $B$ to $\mathscr{H}_{i}$ and $\mathscr{L}_{i}$, respectively for $i=1,2$, and put

$$
R=A-\left(A_{1} \oplus A_{2}\right), \quad S=B-\left(B_{1} \oplus B_{2}\right) .
$$

By Lemma 1 and by the quasidiagonality of $A$ and $B$, the finite-dimensional subspaces $\mathscr{H}_{1}$ and $\mathscr{L}_{1}$ can be chosen so that

$$
V\left(A_{2}\right) \subseteq\left(V_{e}(A)\right)_{\varepsilon}, \quad V\left(B_{2}\right) \subseteq\left(V_{e}(B)\right)_{\varepsilon}
$$

and

$$
\|R\|<\varepsilon, \quad\|S\|<\varepsilon .
$$

Let $I_{\mathscr{H}}$ and $I_{\mathscr{L}}$, be the identity operators on $\mathscr{H}_{j}$ and $\mathscr{L}_{j}$, respectively, for $j=1,2$. The Hilbert space $\mathscr{H} \otimes \mathscr{L}$ decomposes into the orthogonal sum

$$
\mathscr{H} \otimes \mathscr{L}=\bigoplus_{i, j=1}^{2} \mathscr{H}_{i} \otimes \mathscr{L}_{j},
$$

and correspondingly the operator $D=A \otimes I_{\mathscr{L}}-I_{\mathscr{H}} \otimes B=\left[\left(A_{1} \oplus A_{2}\right)+R\right] \otimes I_{\mathscr{L}}$ $-I_{\mathscr{K}} \otimes\left[\left(B_{1} \oplus B_{2}\right)+S\right]$ can be written as

$$
D=\left[\left(A_{1} \otimes I_{\mathscr{L}_{1}}-I_{\mathscr{H}_{1}} \otimes B_{1}\right) \oplus D^{\prime}\right]+R \otimes I_{\mathscr{L}}-I_{\mathscr{H}} \otimes S,
$$

where

$$
D^{\prime}=\bigoplus_{(i, j) \neq(1,1)}\left(A_{i} \otimes I_{\mathscr{L}_{i}}-I_{\mathscr{H}_{1}} \otimes B_{j}\right)
$$


Since for any two operators $T_{1}$ and $T_{2}$ the inclusion $V_{e}\left(T_{1}+T_{2}\right) \subseteq V_{e}\left(T_{1}\right)+V_{e}\left(T_{2}\right)$ holds by the definition of the algebra numerical range, we conclude from (6) and (5) that

$$
V_{e}(D) \subseteq\left(V_{e}\left[\left(A_{1} \otimes I_{\mathscr{L}_{1}}-I_{\mathscr{H}_{1}} \otimes B_{1}\right) \oplus D^{\prime}\right]\right)_{2 \varepsilon} .
$$

Since the essential numerical range of an operator $T$ does not change when $T$ is compressed to a subspace of finite codimension [6], [3, p. 129], (7) implies that $V_{e}(D) \subseteq\left(V_{e}\left(D^{\prime}\right)\right)_{2 \varepsilon}$, hence

$$
V_{e}(D) \subseteq\left(V\left(D^{\prime}\right)\right)_{2 \varepsilon} .
$$

The numerical range of $D^{\prime}$ can be expressed using the identity

$$
V\left(X \otimes I_{\mathcal{N}}-I_{\mathscr{M}} \otimes Y\right)=V(X)-V(Y)
$$

which holds for arbitrary $X \in \mathscr{B}(\mathscr{M})$ and $Y \in \mathscr{B}(\mathscr{N})$, where $\mathscr{M}$ and $\mathscr{N}$ are any Hilbert spaces. This identity follows from [15, p. 137, Corollary 1.3], and it can also be proved directly. (Indeed, the inclusion $V(X)-V(Y) \subseteq V\left(X \otimes I_{\mathcal{N}}-I_{\mathscr{M}} \otimes Y\right)$ can be proved in a similar way as the inclusion (2). The opposite inclusion follows from the obvious relation $V\left(X \otimes I_{\mathcal{N}}-I_{\mathscr{M}} \otimes Y\right) \subseteq V\left(X \otimes I_{\mathcal{S}}\right)-V\left(I_{\mathscr{M}} \otimes Y\right)$ and from the equalities $V\left(X \otimes I_{\mathcal{N}}\right)=V(X), V\left(I_{\mathscr{M}} \otimes Y\right)=V(Y)$, that are well known and easy to see.) Since the numerical range of a direct sum of Hilbert space operators equals the convex hull of the union of numerical ranges of the summands, we have

$$
V\left(D^{\prime}\right)=\operatorname{co}\left[\bigcup_{(i, j) \neq(1,1)}\left(V\left(A_{i}\right)-V\left(B_{j}\right)\right)\right] .
$$

Now it follows from (8), (9), (4) and from the obvious inclusions $V\left(A_{1}\right) \subseteq V(A)$, $V\left(B_{1}\right) \subseteq V(B)$, that

$$
\begin{aligned}
& V_{e}(D) \subseteq\left(\operatorname { c o } \left[\left(V(A)-\left(V_{e}(B)\right)_{\varepsilon}\right) \cup\left(\left(V_{e}(A)\right)_{\varepsilon}-V(B)\right)\right.\right. \\
&\left.\left.\cup\left(\left(V_{e}(A)\right)_{\varepsilon}-\left(V_{e}(B)\right)_{\varepsilon}\right)\right]\right)_{2 \varepsilon} .
\end{aligned}
$$

Let $\varepsilon$ tend to 0 in (10); then (3) follows.

To prove that the inclusion (3) holds without the quasidiagonality assumption, we need

Lemma 2. For any $T \in \mathscr{B}(\mathscr{H})$ and any $\varepsilon>0$ there exists a bounded operator $S$ (acting on some separable Hilbert space) such that the operator $Q=T \oplus S$ is quasidiagonal and

$$
V(Q) \subseteq(V(T))_{\varepsilon}, \quad V_{e}(Q) \subseteq\left(V_{e}(T)\right)_{\varepsilon} .
$$

Proof. The existence of an operator $S$ such that the operator $T \oplus S$ is quasidiagonal is proved by Arveson in [1], so we shall only show that the proof in [1] implies also the inclusions (11). Note first that there exists a compact operator $K$ on $\mathscr{H}$ such that the operator $T^{\prime}=T-K$ satisfies

$$
V\left(T^{\prime}\right) \subseteq\left(V_{e}(T)\right)_{\varepsilon / 2} .
$$


(This can be seen as follows. By Lemma 1 there exists a subspace $\mathscr{H}_{0}$ of finite codimension in $\mathscr{H}$ such that $V\left(T_{0}\right) \subseteq\left(V_{e}(T)\right)_{\varepsilon / 2}$, where $T_{0}$ is the compression of $T$ to $\mathscr{H}_{0}$. Let $P$ be the orthogonal projection onto $\mathscr{H}_{0}$ and $\mu$ any point in $V\left(T_{0}\right)$. Then the operator $K=T-P T P-\mu(I-P)$ satisfies the requirement, since $V(T-K)$ $=V(P T P+\mu(I-P))=V\left(T_{0} \oplus \mu I_{\mathscr{H}_{0}}\right)=V\left(T_{0}\right)$.) By $[1$, pp. 334-335] there exists a sequence of positive finite-rank operators $E_{n}$ on $\mathscr{H}$ and a compact operator $K^{\prime}$, with $\left\|K^{\prime}\right\|<\varepsilon / 2$, acting on the Hilbert space $\mathscr{L}:=\bigoplus_{n=1}^{\infty} E_{n} \mathscr{H}$, such that $T^{\prime}$ is unitarily equivalent to a direct summand of the operator

$$
Q^{\prime}=\left(\bigoplus_{n=1}^{\infty} T_{n}^{\prime}\right)-K^{\prime}
$$

where $T_{n}^{\prime}$ is the compression of $T^{\prime}$ to the subspace $E_{n} \mathscr{H}$ of $\mathscr{H}$. That is, there exist operators $T^{\prime \prime}$ and $S$ such that $Q^{\prime}=T^{\prime \prime} \oplus S$ and such that $T^{\prime \prime}$ is unitarily equivalent to $T^{\prime}$. Since $V\left(T_{n}^{\prime}\right) \subseteq V\left(T^{\prime}\right)$ for every $n$ and since $\left\|K^{\prime}\right\|<\varepsilon / 2$, we have

$$
V\left(Q^{\prime}\right) \subseteq V\left(\bigoplus_{n=1}^{\infty} T_{n}^{\prime}\right)-V\left(K^{\prime}\right) \subseteq\left(V\left(T^{\prime}\right)\right)_{\varepsilon / 2} .
$$

Now we conclude from (12) that $V\left(Q^{\prime}\right) \subseteq\left(V_{e}(T)\right)_{\varepsilon}$. Since $Q^{\prime}=T^{\prime \prime} \oplus S$, this implies in particular that

$$
V(S) \subseteq\left(V_{e}(T)\right)_{\varepsilon} .
$$

Now we put $Q=T \oplus S$. Then $Q$ is a quasidiagonal operator, since it is a compact perturbation of the operator $T^{\prime} \oplus S$ (namely, $T \oplus S=\left(T^{\prime} \oplus S\right)+(K \oplus$ $0)$ ) which is unitarily equivalent to the quasidiagonal operator $T^{\prime \prime} \oplus S=Q^{\prime}$. Finally, we have by (13) and the identities stated in Remark 3

$$
V_{e}(Q)=\operatorname{co}\left[V_{e}(T) \cup V_{e}(S)\right] \subseteq \operatorname{co}\left[V_{e}(T) \cup V(S)\right] \subseteq\left(V_{e}(T)\right)_{\varepsilon}
$$

and

$$
V(Q)=\operatorname{co}[V(T) \cup V(S)] \subseteq \operatorname{co}\left[V(T) \cup(V(T))_{\varepsilon}\right]=(V(T))_{\varepsilon} .
$$

Proof of the inclusion (3) For arbitarary $A \in \mathscr{B}(\mathscr{H}), B \in \mathscr{B}(\mathscr{L})$. Choose any $\varepsilon>0$. By Lemma 2 there exist bounded operators $A^{\prime}$ and $B^{\prime}$ such that the operators $\hat{A}=A \oplus A^{\prime}$ and $\hat{B}=B \oplus B^{\prime}$ are quasidiagonal and such that

$$
\begin{array}{ll}
V(\hat{A}) \subseteq(V(A))_{\varepsilon}, & V_{e}(\hat{A}) \subseteq\left(V_{e}(A)\right)_{\varepsilon}, \\
V(\hat{B}) \subseteq(V(B))_{\varepsilon}, & V_{e}(\hat{B}) \subseteq\left(V_{e}(B)\right)_{\varepsilon} .
\end{array}
$$

Now the operator $D=A \otimes I_{\mathscr{L}}-I_{\mathscr{H}} \otimes B$ is a direct summand of the operator $\hat{D}=\hat{A} \otimes I_{\hat{\mathscr{L}}}-I_{\hat{\mathscr{H}}} \otimes \hat{B}$ (where $\hat{\mathscr{H}}$ and $\hat{\mathscr{L}}$ are the spaces of $\hat{A}$ and $\hat{B}$, respectively), hence $V_{e}(D) \subseteq V_{e}(\hat{D})$. Since $\hat{A}$ and $\hat{B}$ are quasidiagonal, the inclusion (3) holds with $\hat{A}, \hat{B}$ in place of $A, B$; thus (14) implies that

$$
V_{e}(D) \subseteq \operatorname{co}\left[\left(\left(V_{e}(A)\right)_{\varepsilon}-(V(B))_{\varepsilon}\right) \cup\left((V(A))_{\varepsilon}-\left(V_{e}(B)\right)_{\varepsilon}\right)\right] .
$$

When $\varepsilon$ tends to 0 in the last inclusion, we obtain (3).

The proof of the theorem is so completed. As an immediate consequence of the theorem we get that $D_{A B}$ is a compact operator if and only if it is 0 . Indeed, if $D_{A B}$ is compact, then $V_{e}\left(D_{A B}\right)=\{0\}$ and it follows by the theorem that $A=\lambda I=B$ for 
some $\lambda \in \mathbf{C}$. This result can be proved in a more elementary way by the methods of [9]. Indeed, Fong and Sourour characterized in [9] compact elementary operators on $\mathscr{B}(\mathscr{H})$, and this characterization includes as a special case the fact that a nonzero generalized derivation is not compact (see [9, p. 849, Example 1]).

\section{REFERENCES}

1. W. B. Arveson, Notes on extensions of $C^{*}$-algebras, Duke Math. J. 44 (1977), 329-355.

2. F. F. Bonsall and J. Duncan, Numerical ranges of operators on normed spaces and elements of normed algebras, London Math. Soc. Lecture Note Series 2, Cambridge University Press, Cambridge, 1971.

3. __ Numerical ranges. II, London Math. Soc. Lecture Note Series 10, Cambridge University Press, Cambridge, 1973.

4. A. Brown and C. M. Pearcy, Spectra of tensor product of operators, Proc. Amer. Math. Soc. 17 (1966), 162-169.

5. J. B. Conway, A course in functional analysis, Springer-Verlag, New York, 1985.

6. P. A. Fillmore, J. G. Stampfli, and J. P. Williams, On the essential spectrum, the essential numerical range, and a problem of Halmos, Acta Sci. Math. (Szeged) 33 (1972), 179-192.

7. L. A. Fialkow, $A$ note on norm ideals and the operator $X \rightarrow A X-X B$, Israel J. Math. 32 (1979), 331-348.

8. ___ Elements of spectral theory for generalized derivations, J. Operator Theory 3 (1980), 89-113.

9. C. K. Fong and A. R. Sourour, On the operator identity $\sum A_{k} X B_{k}=0$, Canad. J. Math. 31 (1979), 845-857.

10. P. R. Hamos, Ten problems in Hilbert space, Bull. Amer. Math. Soc. 76 (1970), 887-933.

11. D. A. Herrero, Approximation of Hilbert space operators, vol. I, Research Notes in Math. 72, Pitman Press, Boston, Mass., 1982.

12. J. Kyle, Numerical ranges of derivations, Proc. Edinburgh Math. Soc. 21 (1978), 33-39.

13. G. Lumer and M. Rosenblum, Linear operator equations, Proc. Amer. Math. Soc. 10 (1959), 32-41.

14. R. Schatten, Norm ideals of completely continuous operators, Ergeb. Math. Grenzgeb., Band 27, Springer-Verlag, Berlin, 1970.

15. S. Y. Shaw, On numerical ranges of generalized derivations and related properties, J. Austral. Math. Soc. Ser. A 36 (1984), 134-142.

16. J. G. Stampfli, The norm of a derivation, Pacific J. Math. 33 (1970), 737-747.

17. M. Takesaki, Theory of operator algebras. I, Springer-Verlag, New York, 1979.

Department of Mathematics, University of LJubljana, JadransKa 19, 61000 LjublJana, YUGOSLAVIA 